\title{
The burden of orthopaedic disease presenting to a referral hospital in northern Tanzania
}

\author{
Ajay Premkumar ${ }^{1}$, Honest H. Massawe ${ }^{2}$, David J. Mshabaha ${ }^{2}$, Jared R.Foran ${ }^{3}$, XiaohanYing ${ }^{4}$ and Neil P. Sheth $^{4 *}$ \\ ${ }^{1}$ Department of Orthopaedic Surgery, Emory University School of Medicine, Atlanta, GA 30329, USA \\ ${ }^{2}$ Department of Orthopaedic Surgery, Kilimanjaro Christian Medical Center, Moshi, Tanzania \\ ${ }^{3}$ Orthopedic Surgery,Adult Reconstructive Orthopedic Surgery, Golden, CO, Ortho Colorado Hospital, Colorado, USA \\ ${ }^{4}$ Adult Reconstruction,Department of OrthopaedicSugery, Philadelphia VA Medical Center, University of Pennsylvania, Philadelphia, PA, USA
}

\begin{abstract}
Background: In low and middle-income countries, country specific data is scarce regarding the burden of surgical disease, with most estimates extrapolated from indirect methods. Kilimanjaro Christian Medical Center (KCMC) is the only tertiary referral hospital for a population of over 11 million in Northern Tanzania. This study aims to directly quantify the current orthopaedic burden of disease at KCMC and provide a foundation to estimate the magnitude and potential benefit of improving access to orthopaedic surgical care in the northern regions of Tanzania.

Methods: Prospective data was collected during June 2015 for 113 patients admitted to the Orthopaedic Surgery ward at KCMC. Retrospective review of available hospital records for 11,678 patients presenting to the KCMC Emergency Medicine Department, Orthopaedic Clinic, and Orthopaedic Ward over the previous 12 months was also performed to obtain a more complete picture of the burden of orthopaedic disease seen at this tertiary referral center.

Results: KCMC treats an average of 11,172 orthopaedic patients each year. Approximately $57.1 \%$ of these patients are seen as outpatients in clinic, $30.1 \%$ are seen in the emergency department and $12.8 \%$ are admitted as inpatients in the orthopaedic ward. Road traffic accidents (RTAs) represented the most common etiology of injury requiring ward admission at $63.7 \%$, followed by falls at $29.2 \%$, and assaults at $4.4 \%$. Of admissions between ages $15-45$, $73.5 \%$ were from RTAs. The majority of RTAs, $52.8 \%$, involved a motorcycle and $30.56 \%$ involved pedestrians. Femur fractures were the most common injury seen (39.0\%), followed by tibia (27.2\%) and radius $(17.7 \%)$ fractures. Patients used a wide variety of transportation methods and took 2.3 hours on average to arrive at KCMC from the location of their injury. Once in the hospital, patients averaged a length to surgery of 10.2 days and a hospital length of stay of 13.5 days. Of all admissions, $96.5 \%$ had indications for surgical fixation, while only $57.9 \%$ received surgery. $\mathrm{KCMC}$ is the only tertiary referral center for a five state region; however, $65.7 \%$ of patients originated from the same state in which the hospital resides.
\end{abstract}

Conclusions: KCMC sees an approximately identical volume of surgical orthopaedic trauma as a Level 1 Trauma Center in the United States, but has significantly fewer material and intellectual resources to meet this surgical burden. These data give a more complete picture of the patient demographics, mechanisms of injury, types of injury and patient outcomes for similar resource-limited locations.

\section{Introduction}

Globally, injuries represent the major cause of death among persons between 1 and 40 years of age [1]. Road Traffic Accidents (RTA) comprise the largest sub-category of all injuries and are responsible for over 1.2 million deaths annually and represent the third leading cause of disability for people aged 15-44 [2,3]. Orthopaedic surgical treatment, which is often indicated for these traumatic injuries, is often poorly accessible in the world's developing countries.

Tanzania, an East African country with a gross domestic product (GDP) per capita of \$998.1, which ranks 202 out of 229 nations worldwide, is no exception to these trends; RTAs were the $4^{\text {th }}$ leading cause of death in Tanzanians aged 14-49 [3,4]. While there is reasonable available data regarding RTA injuries, an untold number of other serious orthopaedic injuries go untreated or undertreated in this region. Inadequate orthopaedic care for such injuries leads not only to death, but also to tremendous long-term disability and lost production for society that is more difficult to quantify.

In Tanzania, a consultant orthopaedic surgeon is a surgeon who has been in practice for at least 5 years following their formal residency training. For a population of almost 50 million people, there are 15 practicing consultant orthopaedic surgeons in Tanzania, of which nine have completed a 4-year orthopaedic surgery residency following medical school and six are general surgeons by-trade. Thus there is one consultant orthopaedic surgeon for every 3.3 million Tanzanians. In Comparison there is one orthopaedicsurgeon for every 11.4 thousand Americans [5]. Given this severe shortage of trained orthopaedic surgeons in Tanzania, most of the surgeons are inundated with acute or neglected trauma and musculoskeletal infections; elective procedures are infrequently performed.

Kilimanjaro Christian Medical Center (KCMC) is an 800-bed facility and the only tertiary referral hospital for a population of roughly

Correspondence to: Neil P. Sheth, Assistant Professor of Orthopaedic Surgery, Adult Reconstruction, Department of OrthopaedicSugery, Philadelphia VA Medical Center, University of Pennsylvania, Philadelphia, PA, USA, Tel: (215) 829-2231; E-mail: neilshethmd@gmail.com

Received: October 18, 2015; Accepted: November 16, 2015; Published: November 20, 2015 
15 million. There are five full-time equivalent consultant orthopaedic surgeons for this population, two of whom work at KCMC. As the largest hospital of five Northern Tanzanian regions, KCMC receives the majority of surgical trauma patients injured in Northern Tanzania [6].

Because of the severe shortage of orthopaedic resources, efforts are currently underway to develop a center for excellence in orthopaedic care at KCMC. This center will be supported by volunteer surgeons at leading international academic institutions who will work with local doctors to provide year-round surgical services, specialty training to local doctors, sustainable access to affordable, high quality surgical care, and a mechanism to address post-operative complications.

In low and middle income countries (LMICs), country specific data is scarce regarding the burden of surgical disease, with most estimates extrapolated from indirect methods, such as demographic surveillance systems, household surveys and a combination of models $[7,8]$. This study aims to directly quantify the current orthopaedic burden of disease at KCMC and provide a foundation to estimate the magnitude and potential benefit of improving access to surgical care in the northern region of Tanzania. The information contained in this study could also guide the implementation of a pre-hospital emergency response system to reduce the burden of trauma in this region $[9,10]$.

\section{Materials and methods}

\section{Study design}

We prospectively collected data for all patients admitted to the Orthopaedic Surgery ward at KCMC in June 2015. In order to get a larger sample size to supplement the prospective data and better quantify the burden of disease at this tertiary referral center, we also performed a retrospective review of all available records over the previous 12-months for patients presenting to the KCMC emergency medicine department, orthopaedic clinic, and orthopaedic ward

This study received KCMC ethics committee approval prior to data collection and analysis.

\section{Setting}

KCMC is located in northern Tanzania and is one of the country's four large tertiary referral centers. It treats patients from the surrounding Arusha, Kilimanjaro, Tanga, Singida and Manyara regions, which has a collective population of over 11 million. However, KCMC has limited material and intellectual resources, including just two full-time equivalent orthopaedic surgeons, 17 orthopaedic residents, 16 trained nurses and three nurses per shift. There is one full-time anesthesiologist and 11 nurse anesthetists who are shared between all departments and five operating rooms. There is one fulltime orthopaedic operating room and a second room shared with the general surgery and Obstetrics and Gynecology Departments and used roughly twice weekly for septic orthopaedic cases. The orthopaedic ward has 57 beds in nine rooms, but often has many more than 57 admitted patients at one time, with patients regularly overflowing from full rooms into narrow corridors.

There is no established pre-hospital emergency response system in this region of Tanzania, and most patients reach the hospital via their own transportation, with ambulances typically reserved for interfacility transfer of critically ill patients.

\section{Data collection}

Prospective data: All patients admitted to the orthopedic ward over a 4-week period were included in prospective data collection, with the exception of patients with isolated spine pathology, as there are limited spine operations performed at KCMC and these patients are typically treated conservatively in the hospital for several weeks to months.

Retrospective data: The KCMC emergency department, orthopaedic clinic, and orthopaedic ward maintain separate and multiple logbooks for every patient presenting for care. A review of all available logbooks regarding patients presenting within the past year to KCMC was conducted by two authors (AP and DM). Ten percent of patient entries were reviewed independently by both authors in order to assess the inter-observer reliability of the retrospective data abstraction.

\section{Outcome measures}

Prospective data was collected on demographics, diagnoses, injury mechanisms, distance travelled to KCMC, prior hospitalizations, and hospital length of stay for orthopaedic ward admissions over a 4-week period in June 2015.

Retrospective review of orthopaedic ward and operating room data examined the number of ward admissions, diagnoses, and discharge status (including deaths). Deaths were corroborated with hospital mortality reports and death certificates. Review of orthopaedic clinic data assessed the total number of clinic patients seen and the percentage with health insurance, as well as the cost incurred by each patient. Review of emergency department records determined the number of visits, orthopaedic consults, and road traffic injuries. All sums of money were presented as United States dollars, using an exchange rate of 2,000 Tanzanian shillings (Tsh) to one US dollar, and adjusted for 2010 purchasing power parity.

\section{Statistical analysis}

Statistical analysis was performed using the R: a language and environment for statistical computing ( $\mathrm{R}$ Foundation for Statistical Computing, http://www.R-project.org). Student $t$-tests were performed for continuous data and $\chi^{2}$ or Fisher exact tests were performed for categorical data, as appropriate. Two-tailed $p$ values $<0.05$ were considered statistically significant. Cohen's Kappa statistic was calculated to assess inter-observer agreement of retrospective data abstraction.

\section{Results}

\section{Prospective cohort}

During our prospective data collection period, excluding primary spine pathology, 115 patients were admitted to the orthopaedic ward. Two patients were unable to provide personal histories due to altered mental status and thus were excluded from the analysis. The remaining 113 patients were identified and prospectively followed during the data collection period.

Of patients admitted during the month of June 2015, the three most common occupations include farmers $(36.8 \%, 43)$, students $(20.5 \%, 24)$ and professional drivers $(11.1 \%, 13)$. Over $95 \%$ of employed patients who had completed their education listed occupations that necessitated robust function of their lower extremities, included occupations such 
as raising livestock, construction and extraction services, maintenance and installation services, and protective services, among others.

\section{Transportation to the hospital (prospective data)}

Utilizing the prospective analysis, of all admissions during June $2015,69.6 \%$ (70) patients were seen at other clinics or hospitals and referred to KCMC. $15.8 \%$ (11) of these patients were seen at two other clinics or hospitals before referral to this tertiary center. All of these patients cited a need for primary orthopaedic care and a lack of resources at the outside hospital as their reason for referral. For those who came straight to the tertiary center, $40.9 \%$ (32) stated that proximity was the primary reason for choosing KCMC, while $33.8 \%$ (27) cited that hospital reputation was most important, and the remaining $25.0 \%$ (20) came to see a specific doctor or receive specific services that were only offered at KCMC.

While $67.3 \%$ (76) of patients arrived to KCMC on the same day of their injury, the average time between injury and arrival to KCMC was two days. Patients traveled 2.3 hours on average to get to KCMC, with one patient traveling up to 14 hours. $39.8 \%$ (45) of patients had greater than two-hour travel time to KCMC.

Of the patients that came straight to KCMC, $48.1 \%$ (38) arrived via a private automobile, $14.2 \%$ (11) arrived via taxi, and $13.2 \%$ (10) arrived via a bus. Less than $6 \%$ of patients arrived directly from their injury site

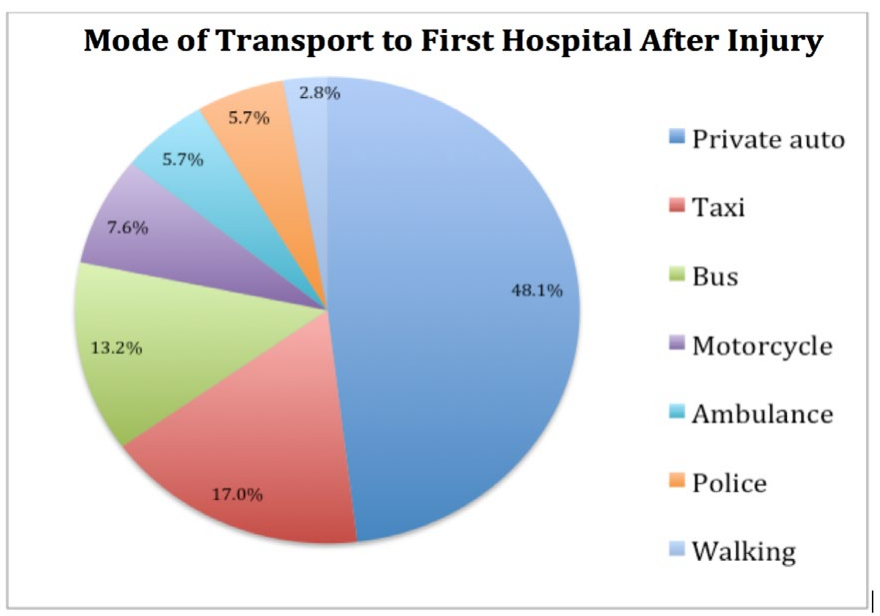

Figure 1. Mode of transport to first hospital after injury.

\section{Mode of Transport Between Facilities}

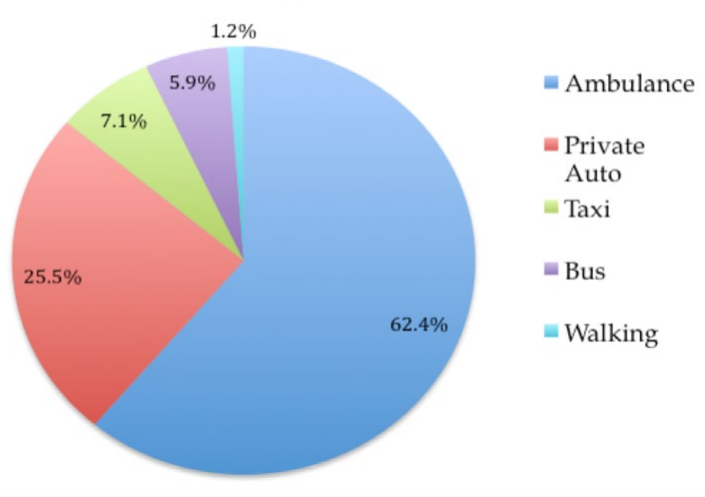

Figure 2. Mode of transport between facilities.

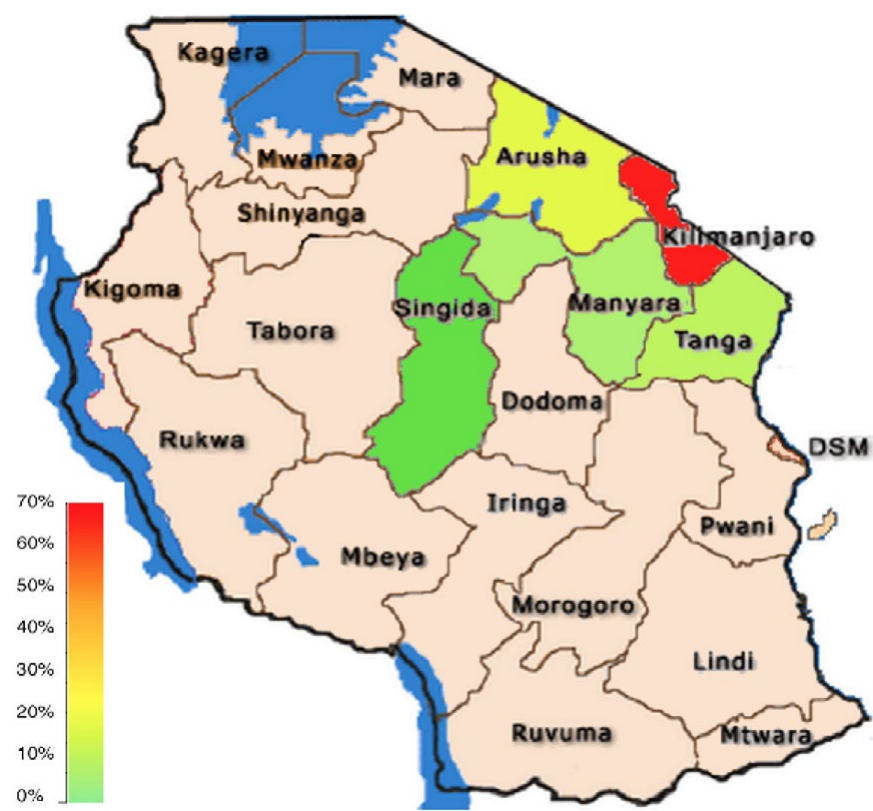

Figure 3. Patient origin by state.

Of the five states which refer patients to KCMC, $65.7 \%$ (898) of patients came from the state of Kilimanjaro, where the hospital is located, $12.7 \%$ (174) came from Arusha, $6.4 \%$ (87) from Tanga, $5.9 \%$ (80) from Manyara and $1.5 \%$ (20) from Singida. While Tanga has a population of roughly 2.1 million, each of the other four regions have a similar population size between 1.4 and 1.7 million inhabitants.

to KCMC by ambulance. A full outline of mode of hospital transport can be seen in Figure 2 and Table 2. For patients transferred to KCMC from other facilities, $62.4 \%$ (44) arrived by ambulance, $23.5 \%$ (16) by private automobile, and $5.9 \%$ (4) by taxi. A more complete picture of the mode of inter-facility transport can be seen in Figure 3 and Table 3.

\section{Injury mechanism (prospective data)}

RTAs represented the most common etiology of injury requiring admission to the orthopaedic ward during June 2015 at $63.7 \%$ (72), followed by falls at $29.2 \%$ (33) and assaults at $4.4 \%$ (5) (Table 4). Of admitted RTAs, $56.4 \%$ (41) involved vehicular passengers and $43.6 \%$ (31) involved drivers. $52.78 \%$ (38) of accidents related to motorcycles and $30.56 \%$ (21) involved pedestrians. Of pedestrian injuries, $57.1 \%$ (12) involved motorcycles. Over half, 55.3\% (21), of all motorcycle related injuries involved motorcycle taxis, referred to locally as "bodabodas," with the rest related to private motorcycles.

The mean age for those with RTAs was significantly lower at 34.5 (SD 15.1), compared to non-RTA admissions at 42.7 (SD 25.9), $(p=0.04)$. Specifically, $73.5 \%$ (50) of those admitted between the ages of $15-45$ were road injury victims, compared to $35.1 \%$ (13) of those greater than 45 and $21.4 \%$ (3) of those less than 15 , respectively $(\mathrm{p}=<0.0001)$. There was no significant difference between males and females in the likelihood of being involved in a RTA and requiring admission at KCMC during June 2015.

\section{Injury location and type (prospective data)}

The three most common injuries seen during June 2015 in the orthopaedic ward were femur $(39.0 \%$, [53]), tibia (27.2\%, [37]), and radius $(17.7 \%,[22])$ fractures. A complete description of injury location and fracture type can be seen in Table 5. 30.1\% (34) patients had multiple injuries requiring orthopaedic care and $86.7 \%$ (98) of 
patients had lower extremity involvement. 93.2\% (105) had isolated unilateral injuries and 7.8\% (8) had bilateral injuries $(\mathrm{p}=0.174) .3 .5 \%$ (4) had bilateral lower extremity injuries. In total, this equates to 164 injuries in the cohort of 113 patients.

\section{Patient disposition and outcomes (prospective data)}

Of the 113 patients prospectively followed in June 2015,73 patients were discharged home, two died, and 38 patients were still inpatients at the end of our data collection period. 96.5\% (109) had either open fractures, closed comminuted long bone fractures, or peri- or intraarticular fractures, and thus had injuries for which surgical fixation were indicated. Of these 109 patients, 57.9\% (63) received surgery during the data collection period. Of the remaining 46 patients, $34.9 \%$ (38) received skeletal traction as the primary treatment for their injury, while $7.3 \%$ (8) received closed reduction and casting as definitive treatment.

Of the patients treated surgically, 20.6\% (13) received surgery within one day of admission, $50.8 \%$ (32) received surgery within seven days of admission, and $74.6 \%$ (47) received surgery within 14 days of admission. The average time after admission until surgery was 10.2 days. Of the 73 patients discharged during the prospective study period, the average and median length of hospital stay was 13.5 days and 10 days, respectively. There was no significant difference in length of hospital stay for those who received surgery compared to those who did not $(\mathrm{p}=0.12)$.

\section{Retrospective cohort}

A total of 11,678 patients were identified and analyzed through retrospective review. This total comprised 1,427 orthopaedic ward, 3,259 outpatient clinic, and 6,992 emergency department records. Orthopaedic ward records and emergency department death records were available for one year, whereas clinic records were available for only the most recent four months and other emergency department data available for the most recent three months. The data below has been extrapolated to represent an estimate of the daily, monthly, and yearly expected clinic, ward and emergency department burden. Kappa coefficients of retrospective chart review was 0.95 , indicating excellent inter-observer reliability between the two reviewers.

\section{Orthopaedic ward data (retrospective data)}

Patient demographics: Of the 1,427 patients who were admitted

Table 1. Patient Origin ${ }^{\circ}$.

\begin{tabular}{|c|c|c|}
\hline State of Origin & $\boldsymbol{n}$ & $\mathbf{( \% )}$ \\
\hline Kilimanjaro & 898 & $65.70 \%$ \\
\hline Arusha & 174 & $12.7 \%$ \\
\hline Tanga & 87 & $6.4 \%$ \\
\hline Manyara & 80 & $5.9 \%$ \\
\hline Singida & 20 & $1.5 \%$ \\
\hline Dar e Salaam & 13 & $0.9 \%$ \\
\hline Kenya & 9 & $0.7 \%$ \\
\hline Dodoma & 8 & $0.6 \%$ \\
\hline Mara & 8 & $0.6 \%$ \\
\hline Other & 70 & $5.1 \%$ \\
\hline
\end{tabular}

${ }^{\circ}$ Data source: retrospective review of 1 year of orthopaedic war records
Table 2. Mode of Transportaion to first Hospital after Injury.

\begin{tabular}{|l|c|c|}
\hline Transportation Method & $\boldsymbol{n}$ & $\mathbf{( \% )}$ \\
\hline Private auto & 51 & $48.10 \%$ \\
\hline Taxi & 18 & $17 \%$ \\
\hline Bus & 14 & $13.2 \%$ \\
\hline Motorcycle & 8 & $7.6 \%$ \\
\hline Ambulance & 6 & $5.7 \%$ \\
\hline Police & 6 & $5.7 \%$ \\
\hline Walking & 3 & $2.8 \%$ \\
\hline
\end{tabular}

*1 of these 3 patients was carried to the hospital by others on foot

${ }^{\circ}$ Data source: prospectively collected orthopaedic war data June 2015

Table 3. Mode ofTransport between Facilities.

\begin{tabular}{|l|c|c|}
\hline Transportation Method & $\boldsymbol{n}$ & (\%) \\
\hline Ambulance & 53 & $62.40 \%$ \\
\hline Private auto & 20 & $25.50 \%$ \\
\hline Taxi & 6 & $7.10 \%$ \\
\hline Bus & 5 & $5.90 \%$ \\
\hline Walking & 1 & $1.20 \%$ \\
\hline
\end{tabular}

${ }^{\circ}$ Data source: prospectively collected orthopaedic ward data June 2015

to the orthopaedic ward between June 28, 2014 and June 28, 2015, the mean age was 38.6 years (range, 1 year to 96 years) (SD 21.8), of which $71.2 \%$ (978) were male patients. Admitted males were significantly younger than admitted females, with a mean age of 35.0 (SD 19.1) compared to 45.7 (SD 23.4), $(\mathrm{p}<0.0001)$.

As mentioned previously, KCMC is the only tertiary referral hospital for five regions in Northern Tanzania; however, patients do not come in equal numbers from each of the regions. A map of the distribution of the origin of patients admitted to the KCMC orthopaedic ward can be seen in Figure and Table 1.

Discharge Status (retrospective data): Ward records showed that $60.8 \%$ (835 of 1427) of patients were documented as discharged, $1.8 \%$ (26) were documented dead, $1.7 \%$ (24) documented as absconded, and the disposition of $38.0 \%$ (542) was not documented. Of the patients documented as discharged, the average and median length of stay was 18.5 and 9 days, respectively. These values include patients with spine pathology.

For patients who were documented dead in the orthopaedic ward, the average time from admission to death was 16.7 days. Reviewing hospital mortality reports, $92.3 \%$ of these hospital deaths occurred between the hours of $5 \mathrm{pm}$ and $7 \mathrm{am}$. The hospital mortality rate counting only these documented hospital deaths is $1.8 \%$. This value was not significantly different from what was seen during prospective data collection in June 2015.

Orthopaedic clinic and hospital emergency department data (retrospective data): The Orthopaedic clinic handles orthopaedic outpatients via appointment only, and is open two days each week. Over 49 clinic days, a total of 3,259 patients were seen in clinic, corresponding to a rate of 67 patients per clinic or 532 patients per month. An average of $227(42.6 \%)$ of these patients each month had some form of health 
Table 4. Injury Mechanism ${ }^{\circ}$.

\begin{tabular}{|l|c|c|}
\hline Mechanism & $\boldsymbol{n}$ & $\mathbf{( \% )}$ \\
\hline Road Traffic Injury & 72 & $66.20 \%$ \\
\hline Motorcycle & 38 & $52.80 \%$ \\
\hline Pedestrian & 22 & $30.60 \%$ \\
\hline Private auto & 6 & $8.30 \%$ \\
\hline Taxi & 6 & $8.30 \%$ \\
\hline Fall & 33 & $29.20 \%$ \\
\hline Assault & 2 & $1.80 \%$ \\
\hline Other & 6 & $5.30 \%$ \\
\hline
\end{tabular}

${ }^{\circ}$ Data source: prospectively collected orthopaedic ward data June 2015

Table 5. Fracture Location \& Type ${ }^{\circ}$.

\begin{tabular}{|c|c|c|}
\hline Fracture & $\mathbf{n}$ & $(\%)$ \\
\hline Femur & 53 & $38.89 \%$ \\
\hline Femoral Shaft & 25 & $47.20 \%$ \\
\hline Femoral Neck & 11 & $20.80 \%$ \\
\hline Distal Femur & 6 & $11.30 \%$ \\
\hline Intertrochanteric & 5 & $9.40 \%$ \\
\hline Subtrochanteric & 3 & $5.70 \%$ \\
\hline Femoral Head & 3 & $5.70 \%$ \\
\hline Tibia or Fibula & 37 & $27.38 \%$ \\
\hline Tibia and Fibula & 25 & $67.60 \%$ \\
\hline Tibial Plateau & 6 & $16.20 \%$ \\
\hline Bimalleolar & 3 & $8.10 \%$ \\
\hline Tibia & 2 & $5.40 \%$ \\
\hline Medial malleolus & 1 & $2.70 \%$ \\
\hline Radius or Ulna & 24 & $17.76 \%$ \\
\hline Radius/Ulna & 13 & $54.20 \%$ \\
\hline Distal Radius & 10 & $41.70 \%$ \\
\hline Monteggia & 1 & $4.20 \%$ \\
\hline Humerus & 9 & $6.62 \%$ \\
\hline Humerus Shaft & 6 & $66.70 \%$ \\
\hline Supracondylar & 3 & $33.30 \%$ \\
\hline Hand & 6 & $4.41 \%$ \\
\hline$M C P^{*}$ & 4 & $66.70 \%$ \\
\hline Metatarsal & 2 & $33.30 \%$ \\
\hline Patella & 3 & $2.21 \%$ \\
\hline Foot & 2 & $1.48 \%$ \\
\hline Talar Neck & 1 & $50 \%$ \\
\hline Calcanues & 1 & $50 \%$ \\
\hline Pelvis & 2 & $1.47 \%$ \\
\hline
\end{tabular}

*MCP=metacarphophalangeal joint

${ }^{\circ}$ Data source: prospectively collected orthopaedic ward data June 2015

insurance, while all other patients paid out of pocket for their clinic visit. The majority of patients paying out of pocket paid roughly $\$ 2.50$; however, depending on the services provided, some were required to pay as much as $\$ 25.00$. For comparison, the GDP per capita in Tanzania per week is $\$ 13.46$ [4].

The emergency room at KCMC is referred to as the Hospital's Casualty Bay. Retrospective review of 6,992 chronological Casualty Bay visits between March and June 2015 demonstrated that the Casualty Ward sees an average of 57 patients per day, 1,777 patients per month, or 20,919 patients per year. Of these, $15.8 \%(1,103)$ required consultation with orthopaedic surgery; thus, there were on average 9 patients per day, 280 patients per month, and 3,301 patients per year presenting at the Casualty Bay requiring orthopaedic services. $68.2 \%$ (753) of these patients had injuries related to RTAs. Those with injuries related to RTAs had an average age of 35.6 (SD 16.35).

\section{Discussion}

In 2010, an estimated $32.9 \%$ (16.9 million) of all deaths worldwide were lost from conditions requiring surgical care, a number that surpassed 2010 deaths from HIV/AIDs, TB, and malaria combined. However, the critical need for timely, safe, and affordable basic surgical services in the world's poorest areas is widely ignored [11.12]. The greatest unmet surgical need can be seen in sub-Saharan Africa and south Asiam, and low operative volumes in these regions are associated with high disability and fatality rates [13]. The unmet need for orthopaedic surgical care is no exception to these global trends. Increased motor vehicle access without a paired increase in road safety and emergency response systems will only exacerbate the burden of road-related musculoskeletal disease and ultimately the burden of disability in these regions.

This study sought to describe the current burden of musculoskeletal disease presenting at the largest tertiary referral hospital in Northern Tanzania, a sub-Saharan country. The goal of this work is to clarify the local disease burden, and ultimately help develop and promote local treatment and preventive strategies, inform decision makers for resource allocation, and address issues relating to the local health workforce.

Extrapolating prospective inpatient ward data and available retrospective outpatient clinic and Casualty Bay information, the KCMC orthopaedic department sees approximately 11,172 patients each year. Approximately $57.1 \%(6,384)$ of these patients are seen as outpatients in clinic, $30.1 \%(3,360)$ are seen in the Casualty Bay, and $12.8 \%(1,427)$ are admitted as inpatients in the orthopaedic ward. Our data shows the overwhelming majority of patients present to the hospital with traumatic injuries requiring emergent or urgent treatment and very few patients are provided elective or planned surgical treatment.

For comparison, in 2014, approximately 1,386 orthopaedic trauma cases were documented at the San Francisco General Hospital (SFGH), a Level 1 Trauma Center in the United States [14]. We chose SFGH for comparison because it treated a similar number of orthopaedic trauma cases to $\operatorname{KCMC~(1,308~orthopaedic~trauma~cases)~during~a~}$ similar time period. While the burden of trauma seen at KCMC is almost identical to that seen at SFGH, there was a large disparity in the number of available trained providers to meet this burden: SFGH had 14 fully trained orthopaedic surgeons who specialized in trauma care compared to only 2 full-time equivalent general orthopaedic surgeons at KCMC. Such comparisons highlight the similarities in traumatic disease burden and gross disparities in access to surgical care between hospitals in LMICs and developed countries.

In addition to highlighting RTAs as a leading cause of injury in 
this region, our data also highlights systemic issues with hospital transportation after an injury. There is no pre-hospital emergency response system in Tanzania, and there are limited ambulances available for timely transport to the hospital. Approximately $80 \%$ of patients used a private automobile, taxi or bus to get to the hospital from the location of their injury, with an average travel time of 2.3 hours. While there is an ambulance system for inter-facility patient transfer, its use is far from pervasive. As seen in Figure 1, it is apparent that patients who live farther away from the hospital are less likely to access hospital services. Assuming that the prevalence of injury is roughly similar in all five states which refers trauma to KCMC, it is possible that in more remote regions, patients simply are unable to travel or do not survive the journey to KCMC from their local hospital which does not have any orthopaedic surgeons and appropriate resources to provide definitive musculoskeletal care.

Similarly, almost half of the patients seen in the Orthopaedic outpatient clinic had some form of health insurance, an insurance coverage rate more than double that of the general Tanzanian population. It seems that several patients who do not have insurance may avoid seeking care due to perceived or real costs of doing so. Further analyses should explore whether there is indeed a higher mortality rate from injuries in the more remote regions of the country and how the perceived and real cost of care impacts decisions to access care among the region's poor.

There were several strengths to this study, including its prospective nature and the large sample size under supplementary retrospective review. There are also several limitations to this study, and some that may underestimate the true burden of orthopaedic disease seen at KCMC. For example, during our retrospective review, each of the 26 cases documented as deaths were evaluated and death confirmed from mortality reports. However, this is most likely an underestimate of the true burden of hospital mortality given poor reporting practices and incomplete patient records [1]. In addition, our prospective data collection excluded spine pathology, as there are limited spine procedures performed at KCMC and the standard of care provided to these patients is typically several weeks to months of bed rest, physical therapy, pain control, and in some cases cervical spine skeletal traction. In this light, including spine pathology would increase the total number of patients seen at KCMC as well as significantly increase the average patient length of stay and proportion who did not receive surgery but for whom surgery was indicated. Lastly, our study does not take into consideration the population who does not access allopathic medical institutions in this region. For example, alternative healers are common in Northern Tanzania and other studies in sub-Saharan Africa have shown that up to $85 \%$ of people visit alternative healers (including traditional "bone setters") first after suffering an acute fracture [15]. While there were anecdotal stories from hospital staff that many patients arrive to KCMC after improper treatment by village healers, none of the patients seen at KCMC during June 2015 saw an alternative healer before visiting the hospital. Thus, our data may suggest that a significant segment of the Northern Tanzanian population does not access the allopathic medical system for trauma management. Further work should be undertaken to more precisely discern the potential role of alternative healers in this region, as well as identify bridges for collaboration with these healers and pathways to increase awareness of allopathic medical centers and the services they offer.

In conclusion, the burden of orthopaedic surgical disease seen at a tertiary referral hospital in Tanzania is dominated by trauma, and is roughly similar in magnitude to the trauma seen in a level one trauma center in the United States; however, there are significantly fewer material and intellectual resources to meet this burden in Tanzania and elective cases are typically ignored. Our analysis underscores the importance of road safety interventions, pre-hospital emergency response systems, robust hospital documentation, as well as the need to improve access to timely, safe and affordable care to the region's poor. This analysis will also help provide a foundation for future studies in this region, and can be a launching point for quantifying the unmet need for orthopaedic surgery and musculoskeletal care and the downstream benefits and potential cost of meeting this need.

\section{References}

1. Krug EG, Sharma GK, Lozano R (2000) The global burden of injuries. Am J Public Health 90: 523-526. [Crossref]

2. WHO (2013) Global status report on road safety 2013: supporting a decade of action. Geneva: World Health Organization, 2013.

3. WHO (2004) The global burden of disease: 2004 update. Geneva: World Health Organization, 2004.

4. The World Bank. GDP per capita (current US\$). 2011-14.

5. American Academy of Orthopaedic surgeons. Orthopaedic Surgeon Quick Facts.

6. KiliManjaro Christian Medical Centre.

7. Petroze RT, Nzayisenga A, Calland JF, Ntakiyiruta G (2011) Surgical volumes at the district hospital: a retrospective review of national data in Rwanda. East Cent Afr J Surg 16: 102-111.

8. Weiser TG, Regenbogen SE, Thompson KD, Haynes AB, Lipsitz SR, et al. (2008) An estimation of the global volume of surgery: a modelling strategy based on available data. Lancet 372: 139-144. [Crossref]

9. Jayaraman S, Mabweijano JR, Lipnick MS, Caldwell N, Miyamoto J, et al. (2009) First things first: effectiveness and scalability of a basic prehospital trauma care program for lay first-responders in Kampala, Uganda. PLoS One 4: e6955. [Crossref]

10. M Tiska, M Adu-Ampofo, G Boakye, L Tuuli, C Mock (2004) A model of prehospital trauma training for lay persons devised in Africa. Emerg Med J 21: 237-239. [Crossref]

11. Shrime MG, Bickler SW, Alkire BC, Mock C (2015) Global burden of surgical disease: an estimation from the provider perspective. Lancet Glob Health 3: S8-S9. [Crossref]

12. Lozano R, Naghavi M, Foreman K, Lim S, Shibuya K, et al. (2012) Global and regional mortality from 235 causes of death for 20 age groups in 1990 and 2010: a systematic analysis for the Global Burden of Disease Study 2010. Lancet 380: 2095-2128.

13. Meara JG, Leather AJ, Hagander L, Alkire BC, Alonso N, et al. (2015) Global Surgery 2030: evidence and solutions for achieving health, welfare, and economic development. Lancet 386: 569-624. [Crossref]

14. Mock CN, Adzotor KE, Conklin E, Denno DM, Jurkovich GJ (1993) Trauma outcomes in the rural developing world: comparison with an urban level I trauma center. $J$ Trauma 35: 518-523. [Crossref]

15. Omololu AB, Ogunlade SO, Gopaldasani VK (2008) The practice of traditional bonesetting: training algorithm. Clin Orthop Relat Res 466: 2392-2398. [Crossref]

Copyright: (C2015 Premkumar A. This is an open-access article distributed under the terms of the Creative Commons Attribution License, which permits unrestricted use, distribution, and reproduction in any medium, provided the original author and source are credited. 